\title{
Minimum Shear Viscosity over Entropy Density at Phase Transition?-A Counterexample
}

\author{
Jiunn-Wei Chen, Chang-Tse Hsieh, and Han-Hsin Lin \\ Department of Physics and Center for Theoretical Sciences, \\ National Taiwan University, Taipei 10617
}

\begin{abstract}
The ratio $\eta / s$, shear viscosity $(\eta)$ to entropy density $(s)$, reaches its local minimum at the (second order) phase transition temperature in a wide class of systems. It was suspected that this behavior might be universal. However, a counterexample is found in a system of two weakly self-interacting real scalar fields with one of them condensing at low temperatures while the other remains in the symmetric phase. There is no interaction between the two fields. The resulting $\eta / s$ is monotonically decreasing in temperature despite the phase transition.
\end{abstract}




\section{INTRODUCTION}

What are the most perfect fluids in Nature with the smallest shear viscosity $(\eta)$ per entropy density $(s)$ ? Kovtun, Son, and Starinets (KSS) [1] suspected that they are a class of strongly interacting conformal field theories (CFTs) whose $\eta / s=1 /(4 \pi)$. They even conjectured that $1 /(4 \pi)$ is the minimum bound for $\eta / s$ for all physical systems. Ever since the KSS bound was proposed, much progress has been made in testing this bound and trying to identify the most perfect fluid (see [2, 3] for recent reviews). It is found that $\eta / s$ can be as small as possible (but still positive) in a carefully engineered meson system [4, 5], although the system is metastable. Also, in strongly interacting CFTs, the universal value $\eta / s=1 /(4 \pi)$ is obtained only in the limit of infinite $N$, with $N$ the size of the gauge group, and infinite t'Hooft coupling limit [6]. $1 / N$ corrections can be negative, however, [7, 8] and can modify the $\eta / s$ bound slightly [9, 10].

In the real world, the smallest $\eta / s$ known so far belongs to a system of hot and dense matter thought to be quark gluon plasma just above the phase transition temperature produced at RHIC [11] with $\eta / s=0.1 \pm 0.1$ (theory) \pm 0.08 (experiment) [12]. A robust upper limit $\eta / s<5 \times 1 /(4 \pi)$ was extracted by another group [13] and a lattice computation of gluon plasma yields $\eta / s=0.134(33)$ [14]. Progress has been made in cold unitary fermi gases as well. An analysis of the damping of collective oscillations gives $\eta / s \gtrsim 0.5[15,16]$. Even smaller values of $\eta / s$ are indicated by recent data on the expansion of rotating clouds [17, 18] but more careful analyses are needed [19].

Previous studies have given some clues about where to find the most perfect fluid in nature. The first one is to study strongly interacting systems because strong interaction generally implies small $\eta / s$. The second clue can be found in a large class of systems where $\eta / s$ goes to a local minimum near the phase transition temperature $\left(T_{c}\right)[20,21,25]$. In particular, $\eta / s$ develops a cusp(jump) at $T_{c}$ for a second(first) order phase transition and a smooth local minimum for a cross over. This behavior is seen in QCD with zero baryon chemical potential [20, 21] and near the nuclear liquid-gas phase transition [22]. It is also seen in cold unitary fermi gases [23], in $\mathrm{H}_{2} \mathrm{O}, \mathrm{N}$, and $\mathrm{He}$ and in all the matters with data available in the NIST database [20, 22, 24]. Theoretically, these behaviors can be reproduced in controlled calculations of weakly interacting real scalar field theories [25]. Thus, it was speculated that this feature is universal. If this is indeed the case, then $\eta / s$ can be used to probe some parts of the systems which are hard to explore otherwise. For 
example, one can try to locate the critical point of QCD by measuring $\eta / s[22,26]$.

In this paper, however, we present a counterexample of the $\eta / s$ behavior speculated above. In this model, $\eta / s$ does not go to a local minimum at the second order phase transition temperature. Our model is a mixture of two weakly self-interacting real scalar fields with one condensing at low temperatures while the other remains in the symmetric phase. There is no interaction between the two fields. The advantage of this model is that its $\eta / s$ can be computed reliably as in [25] because of the small couplings [27]. Other counterexamples have been asserted previously in literature. One of them is a $\sigma$ model calculation with a local minimum below $T_{c}$ [28]. In this model, large couplings are used to mimic the case of QCD. Thus, it is not clear this is due to the failure of the Boltzmann equation at large couplings [27], or if the effect is generic. Also, holographic models have constant $\eta / s(=1 / 4 \pi)$ in the limit of infinite $N$ and the infinite 't Hooft coupling limit. If $1 / N$ corrections are added, $\eta / s$ becomes monotonically increasing below $T_{c}$ and a constant above $T_{c}$ [29]. Our model is a field theory model that one can compute directly and reliably. Our final result shows that $\eta / s$ does not have to develop a local minimum at $T_{c}$.

\section{THE MODEL}

We will study real scalar theories in cases I-III:

$$
\begin{aligned}
\mathcal{L}_{I} & =\mathcal{L}_{1}, \\
\mathcal{L}_{I I} & =\mathcal{L}_{2}, \\
\mathcal{L}_{I I I} & =\mathcal{L}_{1}+\mathcal{L}_{2},
\end{aligned}
$$

where

$$
\mathcal{L}_{i}=\frac{1}{2}\left(\partial_{\mu} \phi_{i}\right)^{2}-\frac{1}{2} \mu_{i}^{2} \phi_{i}^{2}-\frac{1}{4} \lambda \phi_{i}^{4}
$$

The $\eta / s$ of cases I and II are well studied in [25] and we follow the treatment there. $\lambda$ and $\mu_{i}^{2}$ are renormalized quantities and the counterterm Lagrangian is not shown. The renormalization condition is that the counterterms do not change the particle mass and the four-point coupling at threshold. We will set $0<\lambda \ll 1$ such that the systems are bounded from below and we can compute to leading order, $\eta$ and $s$, in the $\lambda_{i}$ expansions. In case I, $\mu_{1}^{2}>0$ and $\phi_{1}$ stays in the symmetric phase. The resulting $\eta / s$ is monotonically 


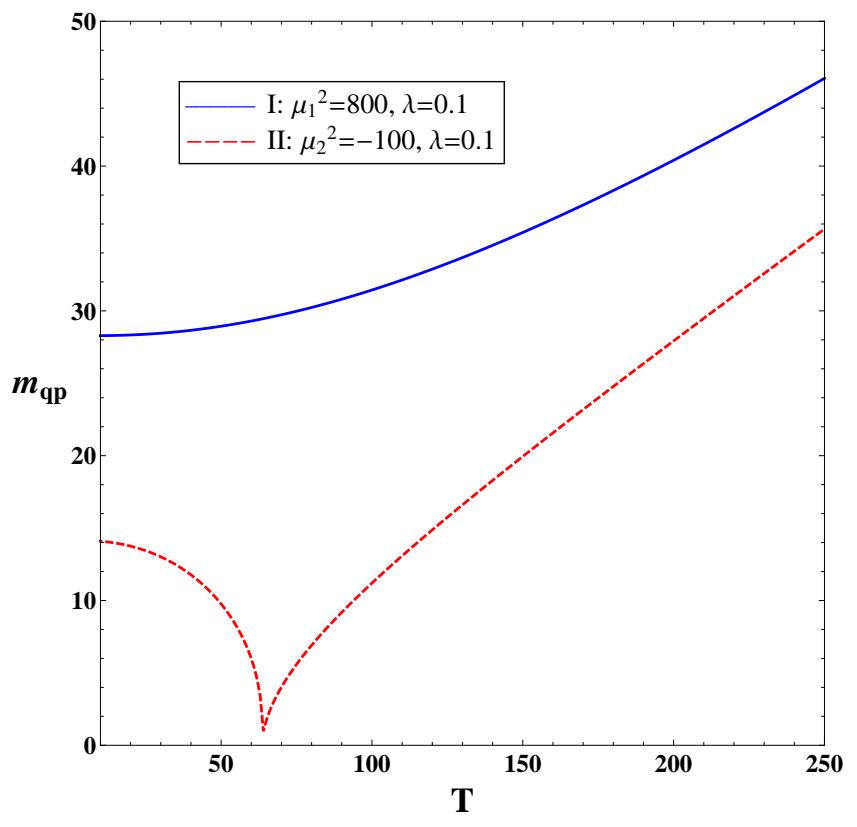

FIG. 1: $m_{q p}$ vs. $T$ for cases I (solid curve, without out symmetry breaking) and II (dashed curve, with symmetry breaking). Parameters can be in arbitrary units.

decreasing in temperature (T) [25]. In case II, $\mu_{2}^{2}<0$. The $\phi_{2} \rightarrow-\phi_{2}$ symmetry is spontaneously broken below the phase transition temperature $T_{c}$. The resulting $\eta / s$ is monotonically decreasing when $T<T_{c}$ and becomes monotonically increasing when $T>T_{c}$. Also, $\eta / s$ forms a cusp at $T_{c}$ under the mean field approximation [25].

Because there is no interaction between $\phi_{1}$ and $\phi_{2}$ in case III, the entropy density is just the sum of the $\phi_{1}$ and $\phi_{2}$ entropy

$$
s_{I I I}=s_{I}+s_{I I} .
$$

Analogously, in a linear response theory, the Kubo formula relates $\eta$ to an ensemble average of a correlator

$$
\eta=-\frac{1}{5} \int_{-\infty}^{0} \mathrm{~d} t^{\prime} \int_{-\infty}^{t^{\prime}} \mathrm{d} t \int \mathrm{d} x^{3}\left\langle\left[T^{i j}(0), T^{i j}(\mathbf{x}, t)\right]\right\rangle
$$

where $T^{i j}$ is the spacial part of the off-diagonal energy momentum tensor. $T_{I I I}^{i j}=T_{I}^{i j}+T_{I I}^{i j}$ and $\left\langle\left[T_{I}^{i j}(0), T_{I I}^{i j}(\mathbf{x}, t)\right]\right\rangle=\left[\left\langle T_{I}^{i j}(0)\right\rangle,\left\langle T_{I I}^{i j}(\mathbf{x}, t)\right\rangle\right]=0$, such that

$$
\eta_{I I I}=\eta_{I}+\eta_{I I}
$$

The high $T$ behavior of $\eta / s$ can be analyzed using the $1 / T$ expansion as in Ref. [22]. By neglect the slow running of the coupling constant, the dimensionful quantities $\mu_{i}^{2}$ and 
$T$ can only contribute to the dimensionless ratio $\eta / s$ through the $\mu_{i}^{2} / T^{2}$ combination (note that it is $\mu_{i}^{2}$, not $\mu_{i}$ that appears in the Lagrangian). As $T \rightarrow \infty, \eta / s$ has the following $1 / T$ expansion

$$
\begin{aligned}
\frac{\eta_{I}}{s_{I}} & \rightarrow \frac{c_{1}}{\lambda^{2}}\left(1+c_{2} \frac{\mu_{1}^{2}}{T^{2}}+\mathcal{O}\left(T^{-3}\right)\right), \\
\frac{\eta_{I I}}{s_{I I}} & \rightarrow \frac{c_{1}}{\lambda^{2}}\left(1+c_{2} \frac{\mu_{2}^{2}}{T^{2}}+\mathcal{O}\left(T^{-3}\right)\right),
\end{aligned}
$$

where $c_{1}>0$ and $c_{2}>0$. There is no $1 / T$ term because as mentioned above, the result does not depend on the sign of $\mu_{i}$. The $1 / T$ expansion of $\eta / s$ in case III has the similar structure

$$
\frac{\eta_{I I I}}{s_{I I I}} \rightarrow \frac{c_{1}^{\prime}}{\lambda^{2}}\left(1+c_{2}^{\prime} \frac{\mu_{1}^{2}+\mu_{2}^{2}}{2 T^{2}}+\mathcal{O}\left(T^{-3}\right)\right) .
$$

Furthermore, in the limit of $\mu_{2}^{2}=\mu_{1}^{2}$, we have $\eta_{I}=\eta_{I I}, s_{I}=s_{I I}$, and $\eta_{I I I} / s_{I I I}=\eta_{I} / s_{I}$ by Eqs.(3) and (5). This implies $c_{1}^{\prime}=c_{1}$ and $c_{2}^{\prime}=c_{2}>0$. Therefore, if $\mu_{1}^{2}+\mu_{2}^{2}>0, \eta_{I I I} / s_{I I I}$ is monotonically decreasing in $T$ as $T \rightarrow \infty$. The question is, whether this behavior persists from large $T$ down to $T_{c}$. Before answering this question numerically, we will try to understand the behaviors of $\eta$ and $s$ separately.

In Fig. 1, we show the typical $m_{q p, i}$, the effective quasiparticle mass of case $i$, as a function of $T$. When $T=0, m_{q p, I}^{2}=\mu_{1}^{2}$ and $m_{q p, I I}^{2}=2\left|\mu_{2}^{2}\right|$. Then $m_{q p, I}^{2}$ increases for increasing $T$ due to the positive thermal mass effect, while $m_{q p, I I}^{2}$ decreases to zero at $T_{c}$, and then increases again at higher $T$. As $T \rightarrow \infty, m_{q p, I}^{2}-m_{q p, I I}^{2}=\mu_{1}^{2}-\mu_{2}^{2}>0$. We have chosen the parameters such that $m_{q p, I}^{2}>m_{q p, I I}^{2}$ at all $T$, which gives $s_{I}<s_{I I}$ in Fig. 2 . The cusp in $s_{I I}$ is barely visible.

In Fig. $2, \eta / T^{3}$ is also shown. Its behavior is very similar to that of $m_{q p}$. To further explore this relation, we use the kinetic theory approximation

$$
\eta \sim \rho v l
$$

where $\rho, v$ and $l$ are the quasiparticle density, velocity and mean free path, respectively. Then using $l \sim 1 / n v \sigma$, where $n$ is the number density and $\sigma$ is the cross section between quasiparticles, we have

$$
\eta \sim \frac{\rho}{n \sigma}=\frac{\epsilon}{\sigma}
$$

where $\epsilon$ is the averaged quasiparticle energy. In a weakly coupled system, $\epsilon$ can be approximated as a gas of free particles with mass $m_{q p}$

$$
\epsilon=\frac{\int d^{3} p \epsilon_{q p} f\left(\epsilon_{q p}\right)}{\int d^{3} p f\left(\epsilon_{q p}\right)}[1+\mathcal{O}(\lambda)],
$$



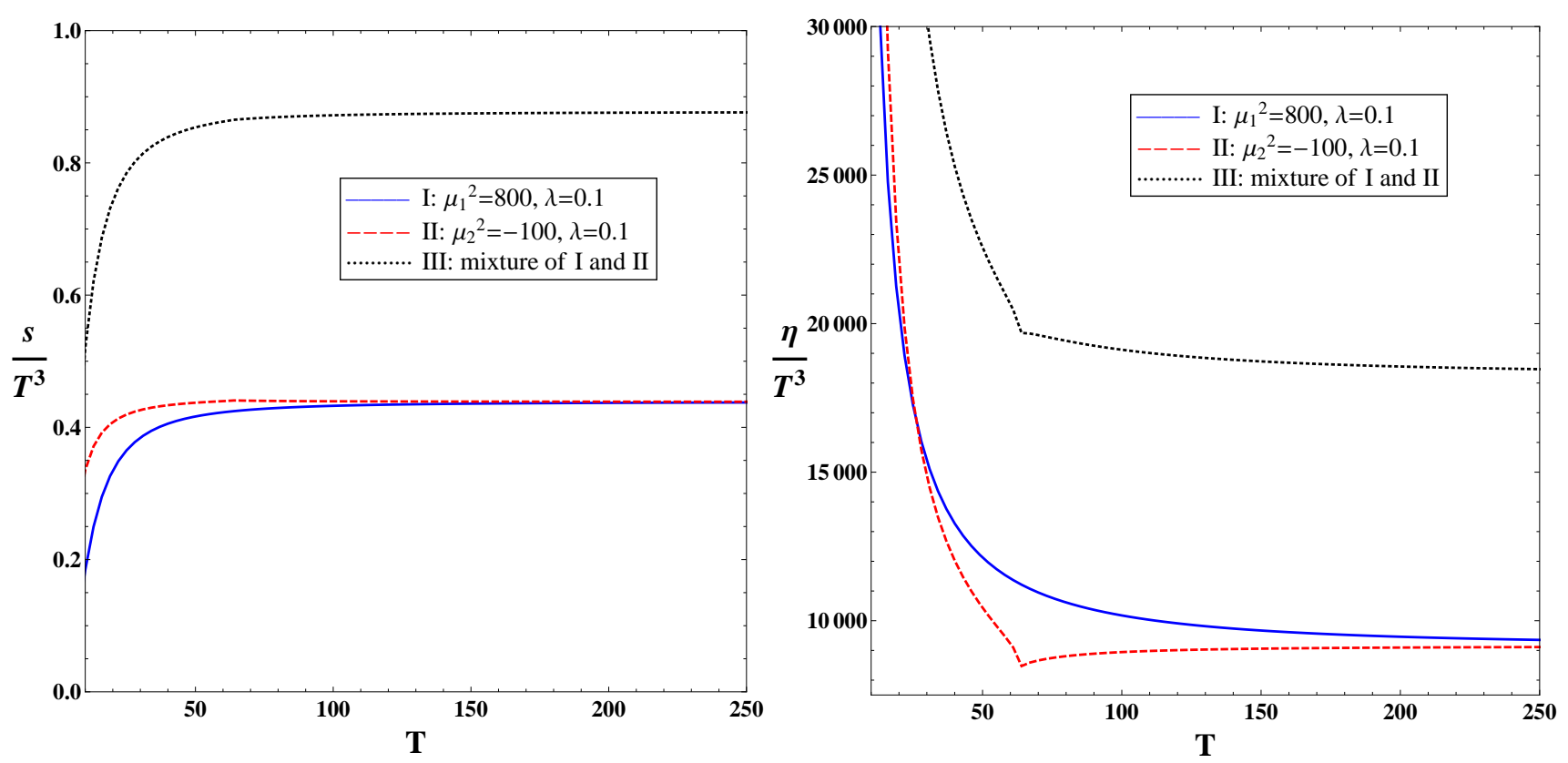

FIG. 2: $s / T^{3}$ and $\eta / T^{3}$ for cases I (solid curve, without out symmetry breaking), II (dashed curve, with symmetry breaking), and III (dotted curve, the mixture of I and II). Parameters can be in arbitrary units.

where $\epsilon_{q p}=\sqrt{p^{2}+m_{q p}^{2}}$ and the Bose-Einstein distribution $f\left(\epsilon_{q p}\right)=1 /\left(e^{\epsilon_{q p} / T}-1\right)$. In two body collisions, $\sigma$ can be approximated as

$$
\sigma \sim \frac{\lambda_{e f f}^{2}}{\epsilon^{2}}
$$

Thus,

$$
\eta \sim \frac{\epsilon^{3}}{\lambda_{e f f}^{2}}
$$

The effective coupling $\lambda_{e f f}$ is $T$ dependent. The explicit expression for the scattering amplitude is [22]

$$
i \mathcal{T} \sim 6 \lambda+(6 \lambda\langle\phi\rangle)^{2}\left[\frac{1}{s-m_{q p}^{2}}+\frac{1}{t-m_{q p}^{2}}+\frac{1}{u-m_{q p}^{2}}\right] .
$$

When $T \sim 0, s \sim 4 m_{q p}^{2}$ and $t \sim u \sim 0$. However, $t \sim u \sim 0$ causes no momentum redistribution and hence the $t$ - and $u$-channels have no contribution to $\eta$. Thus, we can approximate $\lambda_{\text {eff }}$ as

$$
\lambda_{e f f} \sim \lambda+\frac{6 \lambda^{2}\langle\phi\rangle^{2}}{s-m_{q p}^{2}} .
$$

Under this approximation, $\lambda_{\text {eff }}$ decreases smoothly from $2 \lambda$ at $T=0$, to $\lambda$ at $T_{c}$ and stays constant above $T_{c}$. Since $\lambda_{\text {eff }}$ only varies by a factor 2 , we can further approximate 


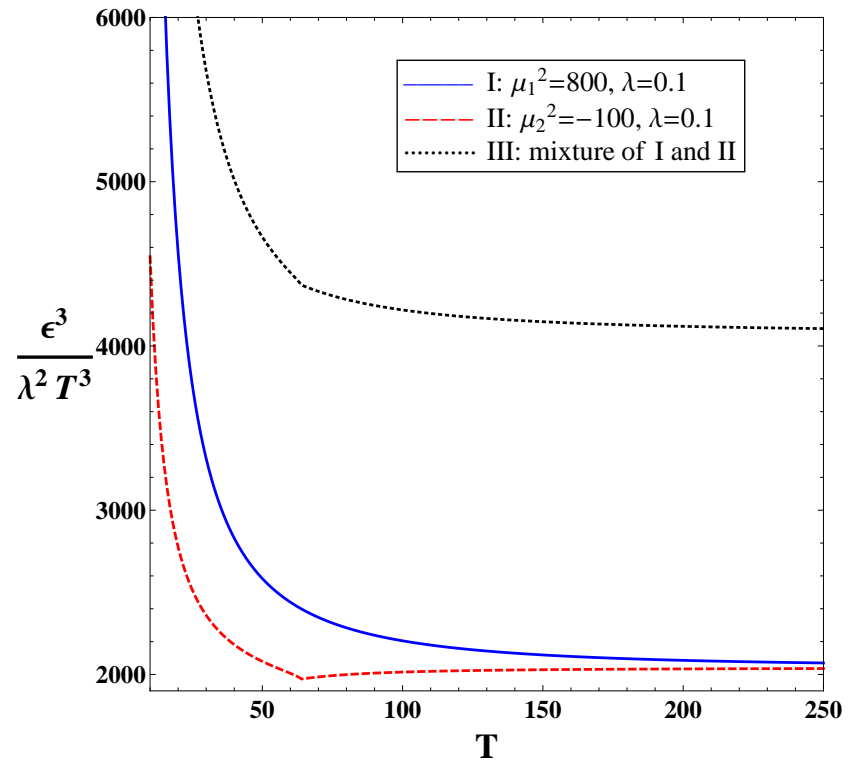

FIG. 3: $\epsilon^{3} /\left(\lambda^{2} T^{3}\right)$ vs. $T$ for cases I (solid curve, without out symmetry breaking), II (dashed curve, with symmetry breaking), and III (dotted curve, the mixture of I and II). Parameters can be in arbitrary units.

it by a constant $\lambda$ such that $\eta \sim \epsilon^{3} / \lambda^{2}$. As shown in Fig. 3, the $T$ dependence of $\epsilon^{3} / \lambda^{2}$ is indeed qualitatively similar to that of $\eta$ in Fig. 2.

Finally, we present the $\eta / s$ results in Fig. 4. They are qualitatively similar to $\eta / T^{3}$. As speculated above, $\eta_{I I I} / s_{I I I}$ is indeed monotonically decreasing both below and above $T_{c}$. This is a counterexample to the previous speculation that $\eta / s$ goes to a local minimum at $T_{c}$ of a second order phase transition.

There are some approximations that we have made in this calculation but none of them should change our conclusion qualitatively. The first one is the "Hartree approximation" that is used to neglect all the sunset diagrams below $T_{c}$. This approximation is good when $T \gg \lambda^{1 / 2} T_{c}$. At lower $T$, the Hartree approximation is not reliable. However, as $T \rightarrow 0, s$ approaches zero exponentially (the excitations are massive) while $\eta$ approaches zero via power laws. As a result, $\eta / s$ is decreasing in cases I-III at low $T$. This feature is not affected despite the Hartree approximation used. The second approximation used is the mean field approximation. Unaccounted quantum fluctuations can make the result reliable in the region $\left|T-T_{c}\right| / T_{c} \lesssim O(\lambda)$. However, this region can be made arbitrarily small by reducing $\lambda$.

Finally, the end point of a first order phase transition (called a critical point $(\mathrm{CP})$ ) is 


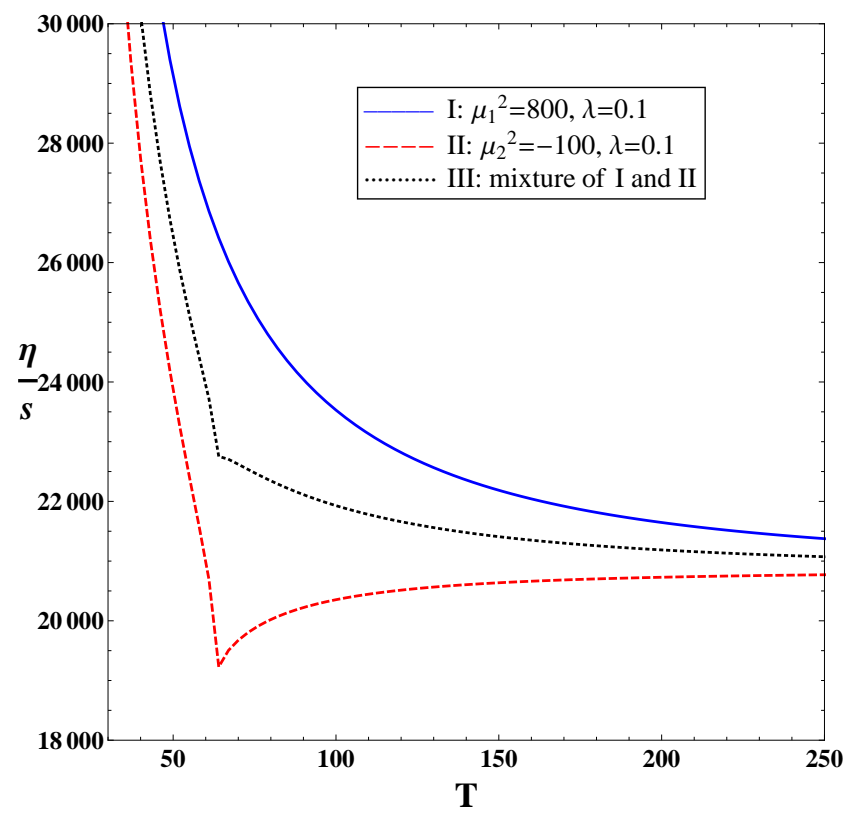

FIG. 4: $\eta / s$ vs. $T$ for cases I (solid curve, without out symmetry breaking), II (dashed curve, with symmetry breaking), and III (dotted curve, the mixture of I and II). Parameters can be in arbitrary units.

also a second order phase transition. This is a special kind of second order phase transition which can be modeled by an effective field theory whose $\phi_{i}^{2}$ and $\phi_{i}^{4}$ couplings vanishes at $T_{c}$, so that the leading coupling is $\phi_{i}^{6}$. Hence the CP case is different from the cases we consider here. In some systems $\eta$ diverges weakly near a CP.

\section{CONCLUSION}

The ratio $\eta / s$, the shear viscosity $(\eta)$ to entropy density $(s)$, reaches its local minimum at the (second order) phase transition temperature in a wide class of systems. It was suspected that this behavior might be universal. However, we have presented a counterexample made of a system of two weakly self-interacting real scalar fields with one of them condensing at low temperatures while the other remains in the symmetric phase. There is no interaction between the two fields. The resulting $\eta / s$ is monotonically decreasing in temperature despite the phase transition.

We thank Brian Smigielski for careful reading of the manuscript. This work is sup- 
ported by the NSC and NCTS of Taiwan.

[1] P. Kovtun, D.T. Son, and A.O. Starinets, Phys.Rev.Lett. 94,111601 (2005).

[2] J. I. Kapusta, arXiv:0809.3746 [nucl-th].

[3] T. Schafer and D. Teaney, Rept. Prog. Phys. 72, 126001 (2009) [arXiv:0904.3107 [hep-ph]].

[4] T. D. Cohen, Phys. Rev. Lett. 99, 021602 (2007) arXiv:hep-th/0702136.

[5] A. Cherman, T. D. Cohen and P. M. Hohler, JHEP 0802, 026 (2008) arXiv:0708.4201 [hep-th]].

[6] D. T. Son and A. O. Starinets, Ann. Rev. Nucl. Part. Sci. 57, 95 (2007) arXiv:0704.0240 [hep-th]].

[7] Y. Kats and P. Petrov, JHEP 0901, 044 (2009) arXiv:0712.0743 [hep-th]].

[8] M. Brigante, H. Liu, R. C. Myers, S. Shenker and S. Yaida, Phys. Rev. D 77, 126006 (2008) arXiv:0712.0805 [hep-th]].

[9] M. Brigante, H. Liu, R. C. Myers, S. Shenker and S. Yaida, Phys. Rev. Lett. 100, 191601 (2008) arXiv:0802.3318 [hep-th]].

[10] A. Buchel, R. C. Myers and A. Sinha, JHEP 0903, 084 (2009) arXiv:0812.2521 [hep-th]].

[11] I. Arsene et al., Nucl. Phys. A 757, 1 (2005); B. B. Back et al., ibid. 757, 28 (2005);

J. Adams et al., ibid. 757, 102 (2005); K. Adcox et al., ibid. 757, 184 (2005).

[12] M. Luzum and P. Romatschke, Phys. Rev. C 78, 034915 (2008) [arXiv:0804.4015 [nucl-th]].

[13] H. Song and U. W. Heinz, J. Phys. G 36, 064033 (2009) arXiv:0812.4274 [nucl-th]].

[14] H. B. Meyer, Phys. Rev. D 76, 101701 (2007), arXiv:0704.1801 [hep-lat].

[15] T. Schafer, Phys. Rev. A 76, 063618 (2007).

[16] A. Turlapov, J. Kinast, B. Clancy, L. Luo, J. Joseph, and J. E. Thomas, J. Low Temp. Phys. 150, 567 (2008).

[17] B. Clancy, L. Luo, J. E. Thomas Phys. Rev. Lett. 99140401 (2007) arXiv:0705.2782 [condmat.other]].

[18] J.E. Thomas, Nucl. Phys. A 830, 665c (2009).

[19] T. Schaefer, C. Chafin, e-Print: arXiv:0912.4236 [cond-mat.quant-gas]; T. Schaefer, e-Print: arXiv:1008.3876 [cond-mat.quant-gas].

[20] L. P. Csernai, J. I. Kapusta and L. D. McLerran, Phys. Rev. Lett. 97, 152303 (2006). 
[21] J. W. Chen and E. Nakano, Phys. Lett. B 647, 371 (2007).

[22] J. W. Chen, Y. H. Li, Y. F. Liu and E. Nakano, Phys. Rev. D76, 114011(2007).

[23] T. Schafer, arXiv:cond-mat/0701251; G. Rupak and T. Schafer, arXiv:0707.1520 [condmat.other].

[24] E.W. Lemmon et al., Thermophysical Properties of Fluid Systems, in NIST Chemistry WebBook, NIST Standard Reference Database Number 69, Eds. Linstrom P.G. \& Mallard, W.G., March 2003 (http://webbook.nist.gov).

[25] J. W. Chen, M. Huang, Y. H. Li, E. Nakano and D. L. Yang, Phys. Lett. B 670, 18 (2008) arXiv:0709.3434 [hep-ph]].

[26] R. A. Lacey et al., Phys. Rev. Lett. 98, 092301 (2007); arXiv:0708.3512.

[27] S. Jeon, Phys. Rev. D 52, 3591 (1995); S. Jeon and L. Yaffe, Phys. Rev. D 53, 5799 (1996).

[28] A. Dobado, F. J. Llanes-Estrada and J. M. Torres-Rincon, Phys. Rev. D 80, 114015 (2009) arXiv:0907.5483 [hep-ph]].

[29] A. Buchel and S. Cremonini, arXiv:1007.2963 [hep-th]. 